\title{
Atmospheric Pressure Plasma Cleaning of Contamination Surfaces Mid-Year Progress Report
}

\author{
Principal Investigators: Gary S. Selwyn and Robert Hicks \\ Los Alamos National Laboratory/University of California, Los Angeles \\ June 1,1997
}

\section{Goals of the Project}

Identify the key physics and chemistry underlying the use of high pressure plasmas for etching removal of actinides and actinide surrogates. Identify key surface reactions and plasma physics necessary for optimization of the atmospheric pressure plasma jet.

\section{Technical Description of the Work}

Decommissioning of transuranic waste (TRU) into low-level radioactive waste (LLW) represents the largest cleanup cost associated with the nuclear weapons complex. This work is directed towards developing a low-cost plasma technology capable of converting TRU into LLW, based upon highly selective plasma etching of plutonium and other actinides from contaminated surfaces. In this way, only the actinide material is removed, leaving the surface less contaminated. The plasma etches actinide material by producing a volatile halide compound, which may be efficiently trapped using filters. To achieve practical, low-cost operation of a plasma capable of etching actinide materials, we have developed a y-mode, resonant-cavity, atmospheric pressure plasma jet (APPJ). In contrast to conventional, low pressure plasmas, the APPJ produces a purely-chemical effluent free of ions, and so achieves very high selectivity and produces negligible damage to the surface. Since the jet operates outside a chamber, many nuclear wastes may be treated including machinery, duct-work, concrete and other building materials. In some cases, it may be necessary to first remove paint from contaminated surfaces using a plasma selective for that surface, then to switch to the actinide etching chemistry for removal of actinide contamination. The goal of this work is to develop the underlying science required for maturation of this technology and to establish early version engineering prototypes.

\section{Accomplishments to Date}

We have made significant progress in this program. The work conducted jointly at Los Alamos and at UCLA. This has been facilitated by exchange of people, equipment and designs between the two locations. The study can be divided into three (3) components: 1) source design, operation and physics, 2) effluent characterization and analysis, 3) surface reactions and decontamination. Some of the key accomplishments in each area are noted below.

\section{1) Source design and physics}

Etching of actinide materials, like most metals, requires the presence of atomic fluorine in order to produce an easily-volatile halide product. One problem this entails is that the 
large quantities of atomic fluorine produced in the plasma source tend to corrode the electrode or wall materials. To solve this problem, a matrix of tests were conducted using different electrode materials, varied radio-frequency power and optical emission monitoring of the APPJ effluent to determine the production of reactive fluorine for each case. The result of this work was the determination that electrodes produced from the alloy monel performed best in terms of both corrosion resistance and the highest production of atomic fluorine. Also, the use of specially-designed slots cut into the center, cylindrical electrode produced about $25 \%$ higher efficiency for production of atomic fluorine (in $\mathrm{He} / \mathrm{Ar} / \mathrm{CF}$, plasmas) and produced comparable benefits for metastable $\mathrm{O}_{2}$ production in $\mathrm{He} / \mathrm{O}_{2}$ plasmas. Metastable $\mathrm{O}_{2}$ is another major product produced by the APPJ and can be used for fast-rate removal of nearly any hydrocarbon contamination. It has no discernible effect on any metal surfaces. All jets designed now at LANL and UCLA utilize these design features.

The figure below shows the data obtained for various electrode materials and $\mathrm{rf}$ power.

\section{O Atom Emission vs RF Power}

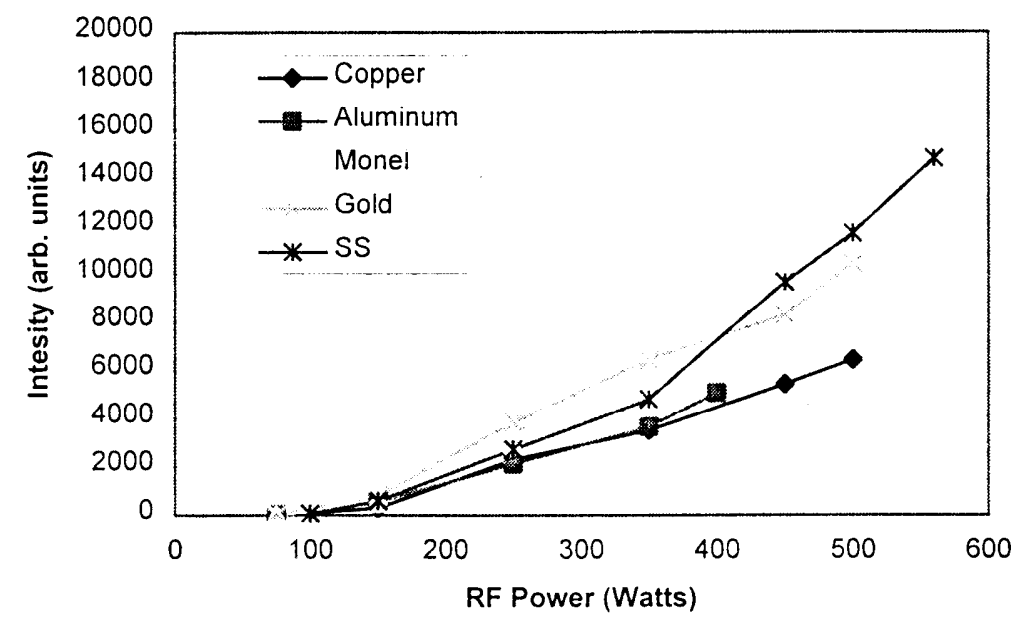

The figure below shows the improvement in fluorine generation provided by the slotted electrode design. 
Fluorine emission vs. RF Power Monel electrode

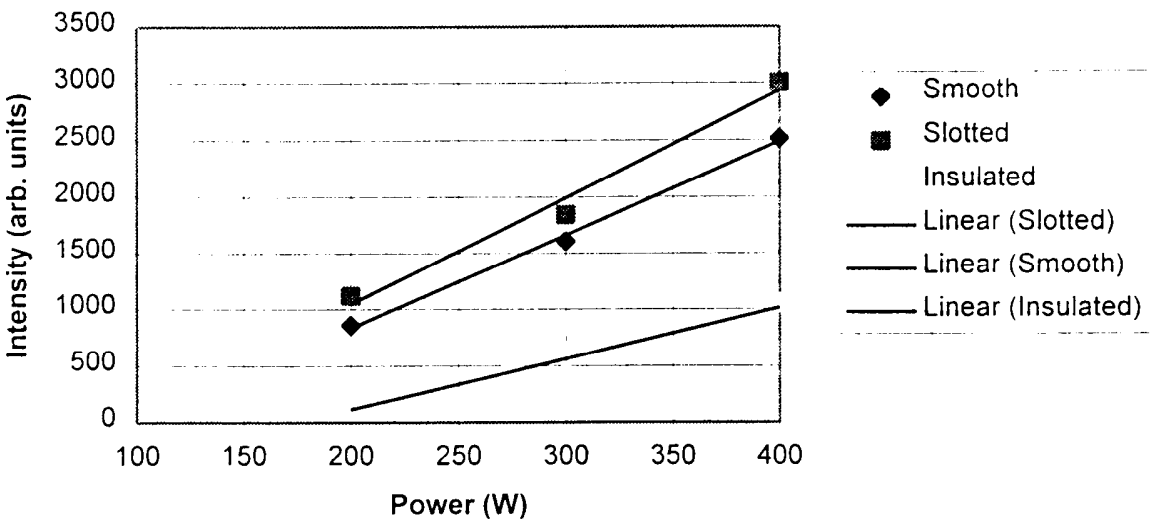

Another improvement in source design has been the use of an inner dielectric sleeve, recently added to the APPJ source for use with Ar and air plasmas. The dielectric sleeve helps prevent arcing and enables the use of higher power density inside the plasma. Also. the dielectric sleeve has proven valuable in the use of other reactive feedgases, such as $\mathrm{He} / \mathrm{Ar} / \mathrm{H}_{2} \mathrm{O}$. This feedstock produces copious amounts of $\mathrm{OH}$ radical that have been shown to be reactive to paint removal. Removal of paint from contaminated surfaces is important because often paint is used to cover surfaces contaminated with $\mathrm{Pu}$. This then requires first removal of the paint (using the water feedstock discussed above), followed by removal of the actinide contamination using the fluorine-containing feed gases.

Very recently we have found that the use of a tubular nozzle placed on the end of the APPJ, not only increases the gas velocity (which helps by thrusting reactive products of the discharge out of the source volume), but also creates an intense hollow-cathode effect. This has the net result of extending the discharge outside of the confines of the jet, producing an even greater density of reactive effluent. Characterization of this nozzle effect is currently on-going, but it is clear that this change will result in further benefit to the APPJ decontamination efficiency.

Study of the basic operation of the APPJ has also led to new insight into the physics of this new discharge. Initially, at low rf power, the discharge starts around the tip of the inner. cylindrical electrode. This is because the electric field is greatest at this region, where the radius of curvature is greatest. Increasing rf power results in no further increase in measured input voltage, instead, the current increases as the discharge moves backwards down the inner electrode. Once the discharge completely encompasses the inner electrode, further increase in rf power results in an increase in applied voltage. This continues until the portions of the cylindrical inner electrode become unevenly hot. This thermionic emission results in arcing. Arcing, of course, is undesirable for two reasons: 1) it can melt the electrode material, 2) it produces a filamentary discharge that is 
inefficient for production of metastables, which are necessary to drive the chemistry of the effluent and the surface chemistry (see next topic in this section).

It is important to understand the arcing mechanism and how to prevent it. Arcing is the usual condition of atmospheric pressure plasmas and special circumstances must be developed in order to prevent arcing. Helium is effective in preventing arcing because it has a low first Townsend coefficient (ionization rate), such that Helium discharges naturally have a high impedance, which limits the current flow through the plasma. This helps limit arcing, due to the high impedance of the plasma. The slots cut into the electrode also help prevent arcing by creating ionization through a hollow cathode effect. That is, electrons produced by secondary electron emission (i.e., ions striking the walls and kicking-off secondary electrons) are trapped between two parallel surfaces (the walls of the slots) at equal and negative potential. Arcs cannot occur between these two surfaces. Finally, the high flow rate helps prevent arcing by providing convective cooling to any hot spots that may develop on the center electrode. As mentioned above, arcing frequently is caused by localized emission of electrons, which reduce the discharge impedance and then increase the local ion current, resulting in a run-away situation.

Changes in the design of the APPJ source have incorporated these newly learned details. We have also used this understanding to partially scale-up the APPJ to a larger size for handling greater power, covering more surface area and providing more thermal stability, though the electrode mass.

\section{Effluent Characterization and Analysis}

Extensive characterization and analysis of the reactive effluent from the APPJ source has been done using optical emission spectroscopy (OES). OES has been used to detail the distribution of reactive species produced by the APPJ (including metastable $\mathrm{O}_{2}$, atomic oxygen, $\mathrm{OH}$ and atomic fluorine). This has been done using a conventional, scanning monochromator fitted with a photomultiplier and with an ultra-high sensitivity, chargecoupled detector (CCD). Maps of the effluent have been drawn, showing the profile of these species as a function of distance from the opening of the APPJ. Until recently, no ions have been detected outside of the APPJ (this is currently under study), however intense ion-based emission is observed inside the plasma jet.

Analysis of the APPJ effluent indicates that within $1 \mathrm{~cm}$ of the opening of the APPJ, there is at first a rapid drop-off in atomic oxygen using a $\mathrm{He} / \mathrm{O}_{2}$ feedgas, whereas the drop-off observed for metastable $\mathrm{O}_{2}$ occurs at a slower rate. Within $3 \mathrm{~cm}$ of the APPJ opening, a correlation is seen for etch rates of polyimide material, with the OES signal observed for metastable $\mathrm{O}_{2}$.

The figure below shows the variation in metastable $\mathrm{O}_{2}$ as a function of distance from the opening of the plasma source. 


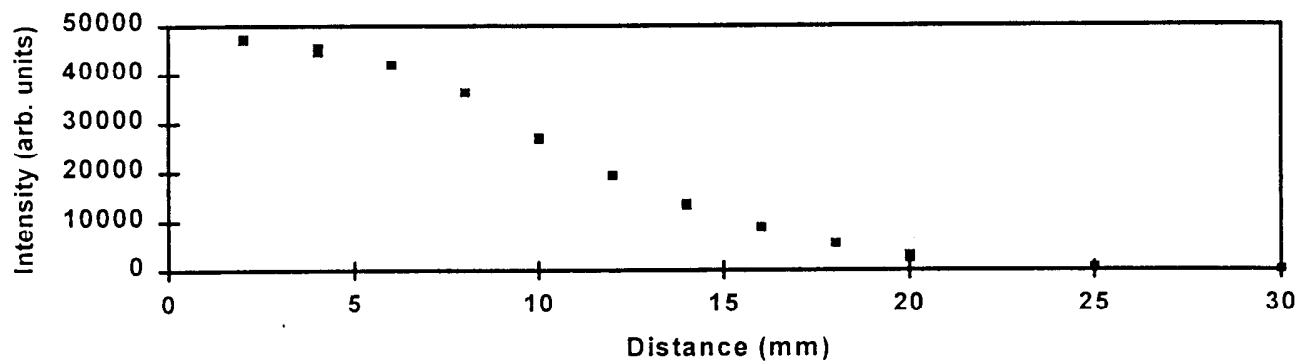

Beyond $3 \mathrm{~cm}$ from the APPJ opening, the metastable $\mathrm{O}_{2}$ has largely been quenched and the signal is nearly absent. However, an increase is seen for the observed emission from atomic oxygen. The signal for atomic oxygen increases up to $10 \mathrm{~cm}$ from nozzle opening; at which point our apparatus is limited for any additional space for measurement. The second increase in atomic oxygen production is attributed to the reaction of He metastables with the oxygen from room air. The signal reaches a minimum at $2 \mathrm{~cm}$ because the mole fraction of the oxygen in the $\mathrm{He} / \mathrm{O}_{2}$ feedstock $(5 \%)$ is much less than that found in air (20\%). So, the signal increases with distance from the nozzle because oxygen from room air gradually infiltrates the stream leaving the jet, then reaction from He metastables leaving the APPJ complete the reaction process.

The figure below shows the observed variation in atomic oxygen, as a function of distance from the opening of the plasma jet.

Oxygen Atom Emission in $\mathrm{O}_{2} / \mathrm{He}$ APPJ with Distance

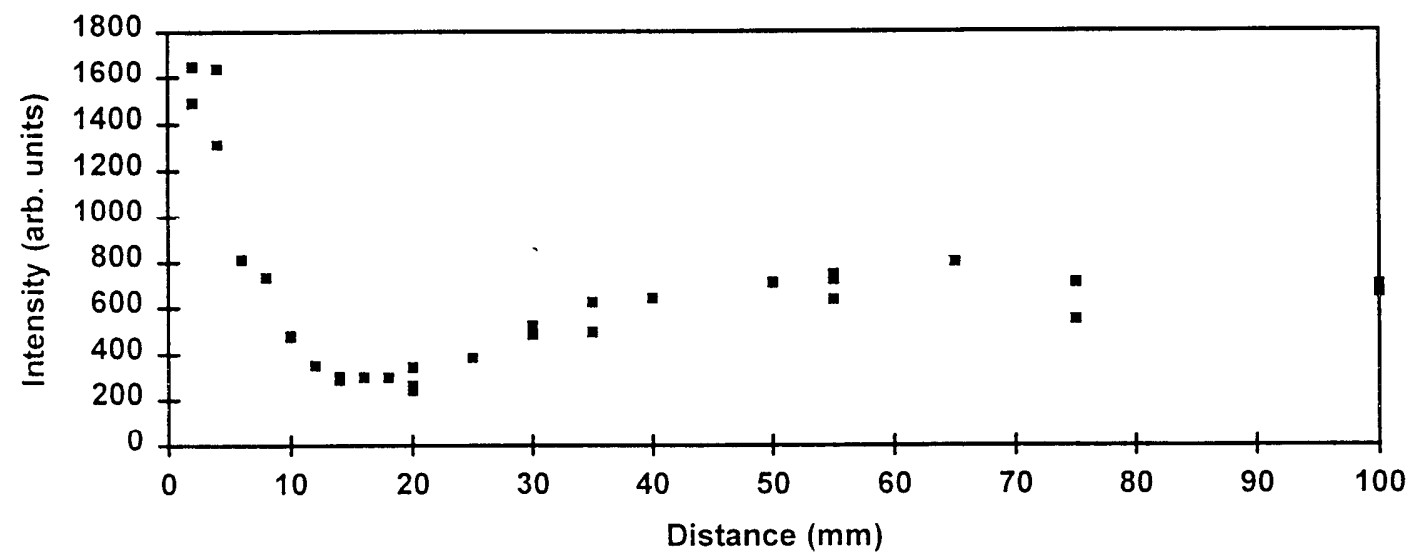

This is highly attractive for use because it indicates that the APPJ may be used at relatively large distances from surfaces for reactive etching of contaminants. Even though the work above was done with $\mathrm{O}_{2}$ from room air, when used in a pipe or duct. the 
flow of $\mathrm{CF}_{4}$ will eventually displace any room air and the local concentration of $\mathrm{CF}_{4}$ will increase. Then, reaction with He or Ar metastables produced the APPJ may be used to convert the $\mathrm{CF}_{4}$ to atomic fluorine. This approach also reduces problems due to corrosion of the jet components exposed to $\mathrm{F}$ atoms. This is because the $\mathrm{F}$ atoms would all be produced downstream of the APPJ. Our current understanding of the chemistry in the plasma effluent is that this is driven by reactions involving rare gas metastables, which then dissociate other molecules (such as $\mathrm{O}_{2}$ or $\mathrm{CF}_{4}$ ) with which they impact. This mechanism works to our benefit by producing species downstream in the effluent, which could not persist long enough outside of the plasma to be of use for the purpose intended.

In addition to OES, temperature measurements have also been made using thermocouples. The temperature of the effluent has been observed typically at below 300 $\mathrm{C}$, but can also be driven higher, even to 600C. The advantages of higher temperature are faster surface reactions, but this must also be reconciled with problems due to burning. Certainly concrete surfaces may be exposed to temperatures as high as 600C. Even at this temperature, the discharge is decidedly non-thermal.

Recent work has also focused on the use of laser measurement methods. notably Raman spectroscopy. The metastable species of $\mathrm{O}_{2}$ have never previously been observed by Raman techniques, but they should be detectable, provided that the concentration is high enough. Recent work has indicated a possible detection of the singlet sigma metastable state using Raman spectroscopy. Preliminary estimates indicate that the concentration of singlet sigma metastable $\mathrm{O}_{2}$ may be as high as 20 Torr in the effluent. The advantage of the Raman measurement method is that it is quantitative, suitable for many species, and unaffected by changes in the electron density and temperature of the plasma.

\section{Surface Reactions and Mechanism}

An etch rates of tantalum, using the output of a $\mathrm{He} / \mathrm{O}_{2} / \mathrm{CF}_{4}$ plasma has been recorded as high as 3 microns/min. Surface analysis of the tantalum metal using XPS shows peaks due to $\mathrm{F} 1 \mathrm{~s}$, Ta 4s, O 1s, Ta 4p, C 1s, Ta 4d and Ta 4f. In addition, deconvolution of the XPS signal shows evidence for $\mathrm{Ta}_{2} \mathrm{O}_{5}$ and $\mathrm{TaF}_{x}, \mathrm{C}-\mathrm{F}, \mathrm{C}=0$, and $\mathrm{C}-\mathrm{O}$ bonds, $\mathrm{CF}_{\mathrm{x}}$ structure and graphitic carbon etch rates of Kapton, a form of polyimide, have been measured over 10 microns/min using the improved designs of the plasma jet. Surface analysis of the Kapton shows an enhancement in the $\mathrm{O}$ signal, roughly the same level of $\mathrm{N}$ signal, and a reduction in the carbon signal after plasma exposure. Based upon the surface analysis and the effluent characterization, it was concluded that metastable $\mathrm{O}_{2}$ is the active species in Kapton etching. Also, paint removal has been demonstrated using $\mathrm{OH}$ generation in a $\mathrm{He} / \mathrm{Ar} / \mathrm{H}_{2} \mathrm{O}$ plasma.

\section{Projections}

Work remaining in FY97 is to complete characterization of the plasma effluent and the composition of the plasma source. We previously developed Raman spectroscopy as a diagnostic tool to aid in this work. Some preliminary results indicate the effluent contains primarily the singlet sigma metastable form of $\mathrm{O}_{2}$, however this requires further 
work and quantification. Further optical emission spectroscopy will be done to complete our survey of the chemical composition of the discharge. Also, we will investigate the use of infrared emission as a means of quantifying the singlet delta state of $\mathrm{O}_{2}$. This state is very difficult to detect, owing its extremely weak emission at $1.227 \mu \mathrm{m}$. We plan to borrow an infrared detector from UCLA for this portion of the work. However, at this time, we anticipate completing our FY97 work by mid-July. This is on schedule with our proposal, which call for underlying science work on the discharge and effluent to be completed in the first year.

\section{Funding}

As of the end of April, 1997, LANL expenditures are approximately $\$ 200 \mathrm{~K}$. This leaves approximately $\$ 17 \mathrm{~K}$, however we received an additional carry-over allocation (from FY96) of $38.2 \mathrm{~K}$. This will be sufficient for work through early July. The project this year will be completed on budget.

Next year we should be able to do better with budgeting because the postdoc planned for work on this project only arrived this week and was originally scheduled for arrival in early March. Work done using a postdoc is much less expensive (by a factor of 4) than work done using TSM assistance. However, since the postdoc was not available (and we had trouble recruiting postdocs in this year of low unemployment), we had to use additional TSM help, which is much more expensive. The new postdoc, Jaeyoung Park. will be working on this project for the next two years.

\section{Issues/Problems}

There are no serious or significant issues to report.

\section{Corrective Actions}

We have received the carry-over allocation from FY96 that we previously reported on. This is now settled without impact to our program.

\section{Additional Information:}

None to report. 